\title{
APPLICATION OF SINGULAR MATRIX BEAMS IN THE SYMMETRICAL PROBLEM OF DEFINITION OF EIGENVALUES
}

\author{
Elena Semenova $^{1}$ Yan Ivakin ${ }^{* 1}$ Elena Frolova ${ }^{1}$ Alena Fomina ${ }^{2}$ Maria Smirnova ${ }^{1}$ \\ ${ }^{1}$ Saint-Petersburg State University of Aerospace Instrumentation, Russian Federation \\ ${ }^{2}$ Central Research Institute of Economy, Management and Information Systems Electronics, \\ Russian Federation
}

The generalized problem of eigenvalue and vectors for singular matrix beams is central in the class of problems of rational construction of computed spectral models of complex modular systems. Solving this problem provides an opportunity to solve these problems, what determined the relevance of this work. The design calculations of complex modular-modular systems have a multivariate character for ensuring their optimal characteristics due to variation within the permissible limits of the elastic-inertial parameters. In the general case such calculations acquire the character of structural-parametric synthesis, when the varied space is supplemented by corrective dynamic devices. The purpose of this article was to provide basic methods for carrying out these calculations. The approach based on the singular decomposition of characteristic matrices was taken as the basis of the research in this paper. This allowed the authors to propose a set of methods for solving this problem, adaptively taking into account the specificity of the available input data. The theoretical significance of the work lies in the development of the modern mathematical and algorithmic apparatus of singular matrix beams, and practical in developing a scientific and methodological basis for solving a corresponding class of applied problems of the dynamics of mechanical and electromechanical systems, for equivalent mathematical and simulation modeling of systems of this class.

Key words: Matrix, Matrix Beam, Eigenvalue Problem, Spec-Central Equation, Characteristic Matrix, Congruence, Dichotomy, Calculator

\section{INTRODUCTION}

For a wide range of applied control problems in complex technical systems related to the study of characteristics and the finding of optimal computational structures of all possible dynamic systems in engineering, seismology, oceanography, quantum chemistry, modern signal processing models and in many other fields of knowledge, the generalized problem of eigenvalues in the form of a solution of the spectral equation $(G-\lambda \Theta) X=0$ for a symmetric real matrix beam $(G, \Theta)$ usually plays the role of a central computational procedure for the solved problems [01, 02]. Moreover, in most typical cases one of the beam arrays or some linear combination of them is positively determined. In such cases, the generalized problem (1) is confidently solved with the use of modern high-quality software implemented in the MATLAB system, which incorporates advanced development experience and actual computer implementation of reliable and efficient numerical methods.

The novelty of the proposed approach to the generalized solution of the symmetric eigenvalue problem for a spectral equation of a given form consists in considering such a solution as a central computational procedure on the basis of the corresponding real, singular matrix beam.

The originality of the solution method proposed by the authors is to realize some preliminary structuring of the initial problem of determining the eigenvalues for the spectral equation, with the goal of exhausting the total zero-space of the calculated matrix beam.

\section{EXPERIMENTAL BASE}

In applied problems of the dynamics of mechanical and electromechanical systems, the rational construction of computed spectral models of complex aggregate-modular systems is based on the use of standardized spectral characteristics of individual building modules. In such cases, the global computational model of the object under study takes the form of a non-free dynamical system with positional links. Similarly, the estimated situation in the study of seismic resistance of complex multi-component building objects. As a potential sample for research, complexes of heterogeneous structures, nodes of engineering networks from several components or subsystems, etc., can also act.

The design calculations of these systems, as a rule, have a multi-variant character for ensuring optimal characteristics of the systems being created due to variation in the allowed elastic-inertial parameters. In the general case, such calculations acquire the character of structural-parametric synthesis, when the varied space is supplemented by correlating dynamic devices.

With the apparent dissimilarity of the problems of the first and second kinds, their model interpretation in the study of spectral characteristics leads to dynamical models of the same structure. 


\section{MATERIALS AND METHODS}

\section{METHOD}

The spectral problem for these systems in the most general situation can be represented as a generalized eigenvalue problem:

$$
(G-\lambda \Theta) X=0
$$

for a singular matrix beam having the following structure:

$$
\begin{aligned}
& G=\left[\begin{array}{c:c:c}
A_{11} & A_{12} & A_{13} \\
\hdashline A_{12}^{*} & \Phi & 0 \\
\hdashline A_{13}^{*} & 0 & 0
\end{array}\right] ; \\
& \Theta=\left[\begin{array}{c:c:c}
E & 0 & 0 \\
\hdashline 0 & 0 & 0 \\
\hdashline 0 & 0 & 0
\end{array}\right],
\end{aligned}
$$

where $\Phi$ and $E$ are - the diagonal and the unit matrix; $A_{12}^{*}$ and $A_{13}^{*}$ are - matrices that are Hermitian conjugate in the general case or symmetric in real problems.

Dimensions of submatrices in the block structure of a quasi-elastic matrix are defined as follows:

$$
\begin{aligned}
& A_{11}: n_{1} \times n_{1} ; \\
& A_{12}: n_{1} \times n_{2} ; \\
& A_{13}: n_{1} \times n_{3} ; \\
& n_{2}<n_{1} ; n_{3}<n_{1}
\end{aligned}
$$

The considered beam structure $(G, \Theta)$ in the general case corresponds to a similar transformation of the starting computational model of the discrete-continuous dynamical system as a result of the spectral decomposition of the initial inertial matrix of the model, [01].

The generalized eigenvalue problem for a symmetric beam in the form (2) is in principle more difficult than the standard problem, since phenomena that substantially complicate the localization of the eigen pairs of the matrix beam can take place. One such phenomenon is generated by the singularity of the beam, which consists in the fact that the characteristic equation of the beam is satisfied for any value of the characteristic exponent. Most often the singularity of a matrix beam is due to the fact that its components have common zero-vectors $X$ such that $G X=\Theta X=0, X \neq 0$. Such vectors are in fact the eigenvectors of the pencil, and any number will be an eigenvalue for them. The uncertainty of the intrinsic spectrum of the beam generated by this phenomenon dictates the necessity of a special initial stage of the analysis of any beam. At this stage, the total zero-space of the matrix pair of the beam is sought and then excluded from the computational model.
Theoretically, this is done by narrowing the beam to the so-called invariant subspace.

In practice, there is also a danger that the beam $(G, \Theta)$ has vectors that are almost annihilated by both beam matrices. In calculations in a discrete computing environment, this leads to the fact that standard programs for solving the generalized eigenvalue problem can calculate some ill-conditioned eigenvalues with pathological properties. These eigenvalues are not only hypersensitive to perturbations of the beam matrices, but their presence in the spectrum significantly lowers the stability of the computational scheme in determining other eigenvalues. Mathematically, in the exact arithmetic of the beam matrices, in the case under consideration there is no common zero-space. In a practical procedure, for a reliable determination of the eigen pairs of a beam, it is also necessary at first to get rid of the "almost-common" zero-space of the beam matrices, setting for this purpose the corresponding criterion for smallness for spectral expansions.

Thus, the eigenvalue problem under consideration requires a special approach, and the use of standard software is possible only after preliminary structuring of the initial model in order to exhaust the common zero-space of the calculated matrix beam.

The solution of the task can be accomplished in two ways. One of them uses the apparatus of singular matrix decomposition, the other - "bordering" the structuring of the calculated beam in combination with an effective algorithm for solving the generalized eigenvalue problem of a bordered beam.

Let us consider the first way of solution [03]. In what follows we use the following notation:

$$
\begin{aligned}
(G, \Theta) \stackrel{P}{\longrightarrow}(\bar{G}, \bar{\Theta}): \bar{G} & =P^{*} G P ; \bar{\Theta}=P^{*} \Theta P \\
P \oplus R & =\operatorname{diag}(P, R)
\end{aligned}
$$

Where $P^{*}$ - the Hermitian conjugate matrix for $P$.

We carry out the orthogonal transformation of the coordinates of the model (1)

$$
X=(U \oplus E \oplus V) Y
$$

and the corresponding beam $(G, \Theta)$ transformation:

$$
\begin{aligned}
& (G, \Theta) \underset{(U \oplus E \oplus V)}{\longrightarrow}(\bar{G}, \bar{\Theta}) \\
& \bar{G}=\left[\begin{array}{c:c:c}
U^{*} A_{11} U & U^{*} A_{12} & U^{*} A_{13} V \\
\hdashline A_{12}^{*} U & \Phi & 0 \\
\hdashline V^{*} A_{13}^{*} U & 0 & 0
\end{array}\right] ;
\end{aligned}
$$


where

$$
\begin{gathered}
\bar{\Theta}=\left[\begin{array}{c:c:c}
U^{*} U & 0 & 0 \\
\hdashline 0 & 0 & 0 \\
\hdashline 0 & 0 & 0
\end{array}\right] \\
U^{*} A_{13} V=\left[\begin{array}{c}
\sigma \\
\hdashline 0
\end{array}\right]
\end{gathered}
$$

$U^{*} U=E$ and $U$ and $V$ are left and right singular matrices for the submatrix, which are orthonormal modal matrices for symmetric products $A_{13} A_{13}^{*}$ and $A_{13}^{*} A_{13}$ respectively; $\sigma$ - the diagonal $n_{3} \times n_{3}$ matrix of the singular numbers of the submatrix $A_{13}$, which are positive square roots of non-zero eigenvalues of the matrices $A_{13} A_{13}^{*}$ and $A_{13}^{*} A_{13}$.

If the submatrix $A_{13}$ has an incomplete rank: $\operatorname{rank}_{13}=\bar{n}_{3}<n_{3}$, its singular expansion takes the form:

$$
U^{*} A_{13} U=\left[\begin{array}{c:c}
\bar{\sigma} & 0 \\
\hdashline 0 & 0
\end{array}\right],
$$

where $\bar{\sigma}$ the diagonal matrix of the order $\bar{n}_{3}$ of non-zero singular numbers of the matrix $A_{13}$ of rank $\bar{n}_{3}$.

In the general case, bearing in mind the possibility of the existence of vectors that are almost annihilated by both beam matrices, it is advisable to represent the matrix $\sigma$ in the form $\sigma=\bar{\sigma} \oplus \Delta,\|\Delta\|=\kappa\|\sigma\|$. Here $k$ is the user criterion of smallness, which allows us to accept $\Delta=0$ and consider the transformed submatrix $A_{13}$ in the form (8). The matrix $\Delta$ will contain zero singular numbers if the submatrix $A_{13}$ has an arithmetically exact incomplete rank.

In accordance with the factorization (8) for the $A_{13}$ submatrix of the transformed beam (7), it is expedient to represent as follows:

$$
\begin{aligned}
& U^{*} A_{11} U=\left[\begin{array}{c:c}
\bar{A}_{11} & \bar{A}_{12} \\
\hdashline \bar{A}_{12}^{*} & \bar{A}_{22}
\end{array}\right] ; \\
& U^{*} A_{12}=\left[\begin{array}{c}
\bar{A}_{13} \\
\overline{\bar{A}}_{23}
\end{array}\right]
\end{aligned}
$$

The submatrices involved in these expressions are characterized by the following dimensions:

$$
\begin{aligned}
& \bar{A}_{11}: \bar{n}_{3} \times \bar{n}_{3} ; \bar{A}_{12}: \bar{n}_{3} \times n_{1}-\bar{n}_{3} ; \bar{A}_{22}:\left(n_{1}-\bar{n}_{3}\right) \times\left(n_{1}-\bar{n}_{3}\right) \\
& \bar{A}_{13}: \bar{n}_{3} \times n_{2} ; \bar{A}_{23}:\left(n_{1}-\bar{n}_{3}\right) \times n_{2} .
\end{aligned}
$$

In accordance with the accepted block representation of matrix submatrices $\bar{G}$, the matrix spectral equation of the transformed beam $(\bar{G}, \bar{\Theta})$ is represented in the following segmented form

$$
\begin{aligned}
& {\left[\begin{array}{c:c:c:c:c}
\bar{A}_{11} & \bar{A}_{12} & \bar{A}_{13} & \bar{\sigma} & 0 \\
\hdashline \bar{A}_{12}^{\bar{z}} & \bar{A}_{22} & \bar{A}_{23} & 0 & 0 \\
\bar{A}_{13}^{*} & \bar{A}_{23}^{\bar{*}} & \overline{1} & 0 & 0 \\
\hdashline \bar{\sigma} & 0 & 0 & 0 & 0 \\
\hdashline 0 & 0 & 0 & 0 & 0
\end{array}\right]\left[\begin{array}{l}
\bar{Y}_{1} \\
\overline{\bar{Y}}_{\bar{Y}} \\
\overline{\bar{Y}}_{3} \\
\overline{\bar{Y}}_{4} \\
\overline{\bar{Y}}_{5}
\end{array}\right]=} \\
& =\lambda\left[\begin{array}{c:c:c:c:c}
E & 0 & 0 & 0 & 0 \\
\hdashline 0 & E & 0 & 0 & 0 \\
\hdashline 0 & 0 & 0 & 0 & 0 \\
\hdashline 0 & 0 & 0 & 0 & 0 \\
\hdashline 0 & 0 & 0 & 0 & 0
\end{array}\right]\left[\begin{array}{l}
\bar{Y}_{1} \\
\overline{\bar{Y}}_{2} \\
\overline{\bar{Y}}_{3} \\
\overline{\bar{Y}}_{4} \\
\overline{\bar{Y}}_{5}
\end{array}\right] .
\end{aligned}
$$

The form (11) of the spectral equation of the transformed beam $(\bar{G}, \bar{\Theta})$ can be called canonical for singular beams with a common zero-space for the matrix components of the beam.

In the case, the beam matrices $(\bar{G}, \bar{\Theta})$ have a common dimension zero space $n_{3}-\bar{n}_{3}$, which generates an infinite set of eigenvalues. Indeed, because of the zero fringing of the matrices of the beam for each vector of the form:

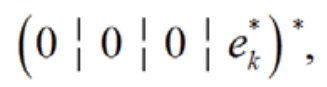

where $e_{k}$ is the unit $\left(n_{3}-\bar{n}_{3}\right)$ component vector, $k=1,2, \ldots, n_{3}-\bar{n}_{3}$, any number is an eigenvalue.

Thus, the beam $(\bar{G}, \bar{\Theta})$, to which the spectral equation of the form (11) corresponds, is singular. In the work program, to solve the generalized eigenvalue problem, there must be a deduction branch, which in this situation exhausts the total zero-space of the matrices of the calculated beam. In the case, this means that the fifth block rows and the column in the expressions (11) for the matrices $\bar{G}$ and $\bar{\Theta}$ must be discarded. After this, solving equations (11) from below upwards, we obtain:

$$
\left.\begin{array}{c}
\bar{\sigma} \bar{Y}_{1}=0 \Rightarrow \bar{Y}_{1}=0 ; \\
\bar{A}_{23}^{*} \bar{Y}_{2}+\Phi \bar{Y}_{3}=0 \Rightarrow \bar{Y}_{3}=-\Phi^{-1} \bar{A}_{23}^{*} \bar{Y}_{Y} ; \\
\left(\bar{A}_{22}-\bar{A}_{23} \Phi^{-1} \bar{A}_{23}^{*} \bar{Y}_{2}=\lambda \bar{Y}_{2} \Rightarrow\left(\bar{A}_{22}-\bar{A}_{23} \Phi^{-1} \bar{A}_{23}^{*}-\lambda E\right) \bar{Y}_{2}=0 ;\right. \\
\bar{A}_{12} \bar{Y}_{2}+\bar{A}_{13} \bar{Y}_{3}+\bar{\sigma} \bar{Y}_{4}=0 \Rightarrow \bar{Y}_{4}=-\bar{\sigma}^{-1}\left(\bar{A}_{12} \bar{Y}_{2}+\bar{A}_{13} \bar{Y}_{3}\right) .
\end{array}\right\}
$$

It follows from the expression in the third line of system (13) that $n_{1}-\bar{n}_{3}$ the finite eigenvalues of a symmetric computational beam $(G, \Theta)$ can be found as a result of solving the standard eigenvalue problem for a symmetric matrix $\bar{A}_{22}-\bar{A}_{23} \Phi^{-1} \bar{A}_{23}^{*}$. The eigenvectors $\bar{Y}_{s}$ of the leading block $4 \times 4$ segment of the canonical shape of the beam corresponding to these values according to the 
dependences (13) have the form

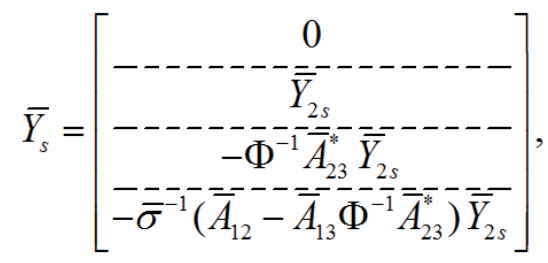

$$
\begin{aligned}
& s=1,2, \ldots, n_{1}-\bar{n}_{3} \text {. }
\end{aligned}
$$

According to the beam segmentation, in the intermediate (7) and canonical (11) forms, the block components of the eigenvectors of these forms are related by the following relations

$$
\begin{aligned}
& Y_{1}=\left[\begin{array}{l}
\bar{Y}_{1} \\
\overline{\bar{Y}}_{2}
\end{array}\right] ; \\
& Y_{2}=\bar{Y}_{3} ; \\
& Y_{3}=\left[\begin{array}{l}
\bar{Y}_{4} \\
\bar{Y}_{5}
\end{array}\right],
\end{aligned}
$$

with $\bar{Y}_{1}=0 ; \bar{Y}_{5}=0$.

We represent singular matrices $U$ and $V$ in block form:

$$
\begin{aligned}
& U=\left[\begin{array}{c:c}
U_{11} & U_{12} \\
\hdashline U_{21} & U_{22}
\end{array}\right] ; \\
& V=\left[\begin{array}{c:c}
V_{11} & V_{12} \\
\hdashline V_{21} & V_{22}
\end{array}\right] .
\end{aligned}
$$

Then the transformation of the eigenvectors $\bar{Y}_{s}$ of the canonical form (11) into the eigenvectors of the original beam (2) can be performed by formulas:

$$
X_{s}=\left[\begin{array}{c:c:c:c:c}
U_{11} & U_{12} & 0 & 0 & 0 \\
\hdashline U_{21} & U_{22} & 0 & 0 & 0 \\
\hdashline 0 & 0 & E & 0 & 0 \\
\hdashline 0 & 0 & 0 & V_{11} & V_{12} \\
\hdashline 0 & 0 & 0 & V_{21} & V_{22}
\end{array}\right]\left[\begin{array}{c}
\bar{Y}_{1} \\
\overline{\bar{Y}}_{2} \\
\overline{\bar{Y}}_{3} \\
\overline{\bar{Y}}_{4} \\
\overline{\bar{Y}}_{5}
\end{array}\right]=\left[\begin{array}{c}
U_{12} \bar{Y}_{2 s} \\
\bar{U}_{22}-\overline{\bar{Y}}_{2 s} \\
-\overline{\bar{Y}}_{3 s} \\
\bar{V}_{11} \overline{\bar{Y}}_{4 s} \\
\hdashline \bar{V}_{21} \overline{\bar{Y}}_{4 s}
\end{array}\right],
$$

where $\bar{Y}_{3 s}$ and $\bar{Y}_{4 s}$ are determined according to the dependences (13).

If the submatrix $A_{13}$ in the block factorization of the pencil has full rank, then the canonical form of the spectral equation for the beam $(\bar{G}, \bar{\Theta})$ will be adequate to the singular leading block $4 \times 4$ segment of equation (11), in which a diagonal matrix $\sigma$ with order $\bar{n}_{3}$ will participate in the diagonal matrix $\sigma$ with order $n_{3}$. Symbolic depen- dencies (13) and (14) will retain their appearance if they replace the name $\bar{\sigma}$ with a name $\sigma$.

In the case, the eigenvalues of the calculated beam are determined as a result of solving the standard eigenvalue problem for a symmetric matrix $\bar{A}_{22}-\bar{A}_{23} \Phi^{-1} \bar{A}_{23}^{*}$. The transformation of the eigenvectors of the nonsingular canonical form of the pencil into eigenvectors of the original beam is realized in the form:

$$
X_{s}=\left[\begin{array}{c}
U_{12} \bar{Y}_{2 s} \\
\bar{U}_{22} \overline{\bar{Y}}_{2 s} \\
-\overline{\bar{Y}}_{3}- \\
-\overline{\bar{Y}_{4}}- \\
\overline{\bar{Y}_{4}}-
\end{array}\right] .
$$

\section{METHODOLOGY}

Let us consider the second way of solving this problem, as some derivative technique from the above described method. In this case, it is expedient to represent the structurization of the calculated matrix beam $(G, \Theta)$ after the exhaustion of the common zero-space in the form:

$$
\begin{aligned}
G & =\left[\begin{array}{cc}
A_{11} & A_{12} \\
A_{12}^{T} & \Phi
\end{array}\right] ; \\
\Theta & =\left[\begin{array}{ll}
E & 0 \\
0 & 0
\end{array}\right],
\end{aligned}
$$

where $\Phi$ - in the general case the degenerate diagonal matrix, some of whose elements or all of its elements are zero.

Let us carry out a similar transformation of the beam under consideration corresponding to the orthogonal coordinate transformation $X=P Y$ :

$$
\begin{aligned}
& (G, \Theta) \stackrel{P}{\longrightarrow}(\bar{G}, \bar{\Theta}): \bar{G}=P^{T} G P ; \\
& \bar{\Theta}=P^{T} \Theta P ; \\
& P=\mu \oplus E,
\end{aligned}
$$

where $\mu$ - the modal matrix of the leading submatrix $A_{11}$ of $n$ order.

The characteristic matrix $K$ of the spectral equation of the transformed beam $(\bar{G}, \bar{\Theta})$ will have the form:

$$
K=\left[\begin{array}{cc}
N-\lambda E & \bar{A}_{12} \\
\bar{A}_{12}^{T} & \Phi
\end{array}\right],
$$

where $N=\mu^{T} A_{11} \mu=\operatorname{diag}\left(v_{i}\right)$ - the diagonal matrix of eigenvalues of the leading submatrix $A_{11}, i=1,2, \ldots, n ; \quad \bar{A}_{12}=\mu^{T} A_{12}, Y=\left(Y_{1}, Y_{2}\right)^{T}$ 
The matrix $K$ is a fringed diagonal $\lambda$-matrix $N-\lambda E$ with a $m$-ordered fringing, $m=n_{2}+n_{3}$. The structural key of this matrix is the matrix pair $\left(T_{n}^{(1)}, \Theta\right)$

$$
\begin{aligned}
& T_{n}^{(1)}=\left[\begin{array}{ll}
N & R^{T} \\
R & \sigma_{0}
\end{array}\right] ; \\
& \Theta=E \oplus 0,
\end{aligned}
$$

which corresponds to a characteristic matrix with one-dimensional fringing.

In expression (22), the bordering parameters of the matrix $T_{n}^{(1)}$ have the following content: $R=\left(d_{1}, d_{2}, \ldots, d_{n 1}\right), \sigma_{0}$ a scalar that is different from zero or equal to zero, depending on the features of the modeled system.

The characteristic matrix $T_{n}^{(1)}-\lambda \Theta$, using the Gaussian factorization for the triangular expansion, can be represented by the following dependences:

$$
\begin{aligned}
& T_{n}^{(1)}-\lambda \Theta=L^{T} \Delta L, \\
& L=\left[\begin{array}{cc}
E & H R^{T} \\
0 & 1
\end{array}\right] ; \\
& H=(N-\lambda E)^{-1}=\operatorname{diag}\left(\frac{1}{v_{i}-\lambda}\right), i=1,2, \ldots, n ; \\
& \Delta(\lambda)=(N-\lambda E) \oplus \Delta_{n+1} ; \\
& \Delta_{n+1}=\sigma_{0}-R H R^{T} .
\end{aligned}
$$

In accordance with the obtained dependences, the diagonal elements $\Delta_{k}$ of the diagonal matrix $\Delta(\lambda)$ in the congruent representation of the matrix $T_{n}^{(1)}-\lambda$ can be written in the form

$$
\begin{aligned}
& \Delta_{k}=v_{k}-\lambda, k=1,2, \ldots, n ; \\
& \Delta_{n+1}=\sigma_{0}-\sum_{i=1}^{n} d_{i}^{2}\left(v_{i}-\lambda\right)^{-1} .
\end{aligned}
$$

On the basis of Sylvester's law of inertia of a symmetric matrix, taking into account Weyl's theorem on the continuity of the eigenvalues of such a matrix, we can state that the number of negative signs of the terms of the sequence $\Delta_{j}, j=1,2, \ldots, n+1$, according to expressions (24) at the current value $\lambda$ is equal to the number of eigenvalues of the matrix beam $\left(T_{n}^{(1)}, \Theta\right)$ that are smaller than $\lambda$. In other words, the sequence $\Delta_{i}, j=1,2, \ldots, n_{1}+1$ can be regarded as a reference sign series for the spectrum $\varsigma(\lambda)$ divisor of the matrix beam $\left(T_{n}^{(1)}, \Theta\right)$ : the number of negative elements $\varsigma(\lambda)$ on the main diagonal of the matrix $\Delta(\lambda)$ is the number of eigenvalues of the beam $\left(T_{n}^{(1)}, \Theta\right)$ that are less than $\lambda$.

The efficiency of the process of localization of the eigenvalues of the beam $\left(T_{n}^{(1)}, \Theta\right)$ can be substantially in- creased if one takes into account the singularities of the characteristic equation of this beam

$$
\sigma_{0}-\sum_{i=1}^{n} d_{i}^{2}\left(v_{i}-\lambda\right)^{-1}=0
$$

It follows from (25) that equation (25) has $n$ for $\sigma_{0} \neq 0$ and $n-1$ for $\sigma_{0}=0$ roots $\lambda_{i}$, which are strictly separated by the eiqenvalues $v_{i}$ of the leading submatrix $N$ of the beam $\left(T_{n}^{(1)}, \Theta\right)$ :

$$
\begin{aligned}
& v_{1}<\lambda_{1}<v_{2}<\lambda_{2}<\ldots<v_{n-1}<\lambda_{n-1}<v_{n}<\lambda_{n}<\infty, \\
& \text { if } \sigma_{0}<0 \\
& 0<\lambda_{1}<v_{1}<\lambda_{2}<v_{2} \ldots<v_{n-2}<\lambda_{n-1}<v_{n-1}<\lambda_{n}<v_{n}, \\
& \text { if } \sigma_{0}>0 \\
& v_{1}<\lambda_{1}<v_{2}<\lambda_{2}<\ldots<v_{n-1}<\lambda_{n-1}<v_{n}, \\
& \text { if } \sigma_{0}=0
\end{aligned}
$$

Conditions (26) make it possible to effectively localize the eigenvalues of the beam $\left(T_{n}^{(1)}, \Theta\right)$ along a parallel computational scheme simultaneously in $n$ or in $n-1$ isolation intervals in accordance with expressions (26). In this case, the divisor of the beam spectrum $\left(T_{n}^{(1)}, \Theta\right)$ is expediently used in the binary modification, as a binary indicator $\xi\left(\Delta_{n+1}\right)$ :

$$
\xi\left(\Delta_{n+1}\right)=\left\{\begin{array}{l}
1 \text { if } \Delta_{n+1}<0 \\
0 \text { if } \Delta_{n+1} \geq 0
\end{array}\right.
$$

The eigenvalue $\lambda_{i}$ in the simplest computational scheme is localized in the interval of the length $\left(v_{i+1}-v_{i}\right) 2^{-k}$ of the $k$ steps of the iteration process, realized by the scheme of dividing the segment in half. In the body of the corresponding work cycle at each $j$-step, the boundaries of the current isolation interval $\left(a_{j}^{(i)}, b_{j}^{(i)}\right)$ for $\lambda_{i}$ are defined as follows:

$$
\left.\begin{array}{c}
\lambda_{i}^{(j-1)}=\frac{1}{2}\left(a_{j-1}^{(i)}+b_{j-1}^{(i)}\right) ; \xi_{j}=\xi\left(\Delta_{n+1}\left(\lambda_{i}^{(j-1)}\right)\right) ; \\
a_{j}^{(i)}=\lambda_{i}^{(j-1)} ; b_{j}^{(i)}=b_{j-1}^{(i)} \quad n p u \xi_{j}=0 ; \\
a_{j}^{(i)}=a_{j-1}^{(i)} ; b_{j}^{(i)}=\lambda_{i}^{(j-1)} \text { npu } \xi_{j}=1,
\end{array}\right\}
$$

where $\lambda_{i}^{(j-1)}, a_{j-1}^{(i)}, b_{j-1}^{(i)}$ - the approximation of the eigenvalue and the boundary of its isolation at the $(j-1)$ step of the iterative process under consideration.

In the general case, instead of the dichotomic scheme considered, localization of the eigenvalues of the beam 
in the intervals in accordance with expressions (26) can use more efficient known procedures [02].

The calculation of the eigenvectors of the beam $\left(T_{n}^{(1)}, \Theta\right)$ can be constructed on the basis of explicit expressions for the components $\alpha_{k s}$ of the eigenvector

$$
\begin{aligned}
& V_{s}=\left(\alpha_{1 s}, \alpha_{2 s}, \ldots, \alpha_{n+1, s}\right) \\
& \alpha_{k s}=-d_{k}\left(v_{k}-\lambda_{s}\right)^{-1}, k=1,2, \ldots, n ; \\
& \alpha_{n+1}=1,
\end{aligned}
$$

where $s \in\left\{\begin{array}{l}{[1, n], \text { если } \sigma_{0} \neq 0} \\ {[1, n-1] \text {, если } \sigma_{0}=0 .}\end{array}\right.$

It can be shown that in special situations associated with the presence of multiple eigenvalues in the beam $\left(T_{n}^{(1)}, \Theta\right)$, the beam structure makes it possible to construct effective and well-conditioned parallel computational schemes for solving the problem of its eigenvalues and vectors [01].

The general algorithm for solving the eigenvalue problem and the matrix-ray vectors $(G, \Theta)$ of the form (19) is realized as a recursive sequence of corresponding problems for beams $\left(T_{n}^{(1)}, \Theta\right)$ of the form (22), accompanied by a consecutive exhaustion of the matrix bordering of the characteristic matrix $K$ of the beam $(G, \Theta)$ [01].

As an illustrative example, let us consider a solution using the method described above for the problem associated with calculating the spectral characteristics of a conservative compound dynamical system with concentrated parameters, composed of three subsystems.

The vectors of the generalized coordinates of the subsystems are taken in the form:

$$
\begin{aligned}
& X_{1}=\left(x_{1}, x_{2}, x_{3}\right)^{*} ; \\
& X_{2}=\left(x_{4}, x_{5}, x_{6}\right)^{*} ; \\
& X_{3}=\left(x_{7}, x_{8}, x_{9}\right)^{*} .
\end{aligned}
$$

Conservative dynamic models of subsystems can be represented in the form

$$
\begin{aligned}
& m_{1}=\operatorname{diag}(3,1,3) ; \quad m_{2}=\operatorname{diag}(2,4,1) ; \\
& m_{3}=\operatorname{diag}(2,2,3) ; \\
& g_{1}=\left[\begin{array}{ccc}
1 & -1 & 0 \\
-1 & 2 & -1 \\
0 & -1 & 1
\end{array}\right] ; \\
& g_{2}=\left[\begin{array}{ccc}
2 & -2 & 0 \\
-2 & 4 & -2 \\
0 & -2 & 2
\end{array}\right] ; \\
& g_{3}=\left[\begin{array}{ccc}
3 & -3 & 0 \\
-3 & 6 & -3 \\
0 & -3 & 3
\end{array}\right] .
\end{aligned}
$$

The reduced values of the quasi-inertial parameters of the nodes and the quasi-elastic parameters of the subsystems are dimensionless, corresponding to certain accepted values of the quasi-inertial and quasi-elastic constants of the calculated dynamic system.

The global system is formed as a connected ensemble of the considered subsystems as a result of superposition of the following links of two types:

- rigid coupling of inertia units 2, 5 and 8

$$
\begin{aligned}
& f_{1}\left(X_{g}\right)=x_{2}-x_{5}=0 ; \\
& f_{2}\left(X_{g}\right)=x_{2}-x_{8}=0 ; \\
& f_{2}\left(X_{g}\right)=x_{5}-x_{8}=0 ;
\end{aligned}
$$

- conjugation of inertial nodes 1, 7 and 3, 6 by elastic connections with dimensionless quasi-elastic coefficients, respectively $c_{1,7}=1 ; c_{3,6}=0,5$;

$$
\begin{aligned}
& f_{3}\left(X_{g}\right)=x_{1}-x_{7}+\delta_{1}=0 ; \\
& f_{4}\left(X_{g}\right)=x_{3}-x_{6}+\delta_{2}=0,
\end{aligned}
$$

where $X_{g}=\left(x_{1}, x_{2}, \ldots, x_{6}\right)^{*} ; \delta_{1}$ and $\delta_{2}-$ the deformations of these elastic joints.

Considering the global system as a non-free dynamical system subordinated to positional constraints of the form (1) and (2), we write the differential equations of motion of the unperturbed global system in a Lagrangian form with multipliers

$$
\left.\begin{array}{c}
M_{0} \ddot{X}_{g}+G_{0} X_{g}=\left(\frac{D F_{1}}{D X_{g}}\right)^{T} \Lambda_{1}+\left(\frac{D F_{2}}{D X_{g}}\right)^{T} \Lambda_{2} ; \\
\frac{D F_{1}}{D X_{g}} X_{g}+\Delta=0 ; \\
\frac{D F_{2}}{D X_{g}} X_{g}=0 \\
C \Delta=\Lambda_{1},
\end{array}\right\},
$$

where

$$
\begin{aligned}
& M_{0}=m_{1} \oplus m_{2} \oplus m_{3} ; \quad G_{0}=g_{1} \oplus g_{2} \oplus g_{3} ; \\
& \Delta=\operatorname{diag}\left(\delta_{1}, \delta_{2}\right) ; C=\operatorname{diag}\left(c_{1,7}, c_{3,6}\right) ; \\
& F_{1}=\left(f_{3}, f_{4}\right) ; F_{2}=\left(f_{1}, f_{2}\right) ; \\
& \frac{D F_{1}}{D X_{g}}, \frac{D F_{2}}{D X_{g}}-\text { Jacobi matrices; } \Lambda_{1}, \Lambda_{2} \text { - indefinite }
\end{aligned}
$$

Lagrange multipliers having the meaning of the reactions of the corresponding bonds, $\oplus-$ the symbol of the direct sum of the matrices. 
Using the extended vector $X_{r}$ of redundant generalized coordinates, the conservative dynamic model of the global system under consideration can be represented in the following matrix-vector form:

$$
\begin{aligned}
& M_{r} X_{r}+G_{r} X_{r}=0, \\
& M_{r}=M_{0} \oplus 0_{4} ; \\
& \text { where } G_{r}=\left[\begin{array}{ccc}
G_{0} & D_{1} & D_{2} \\
D_{1}^{*} & \Phi & 0_{2} \\
D_{2}^{*} & 0_{2} & 0_{2}
\end{array}\right] ; \\
& D_{i}=\left(\frac{D F_{i}}{D X_{g}}\right)^{*}, i=1,2 . \Phi=C^{-1} ; \\
& X_{r}^{*}=\left(X_{g}^{*}, \Lambda_{1}^{*}, \Lambda_{2}^{*}\right) ;
\end{aligned}
$$

$\mathrm{O}_{2}$ and $\mathrm{O}_{4}$ zero matrices of orders 2 and 4 .

Numerical versions of the matrices $M$ and $G$ taking into account the elastic-inertial characteristics of the subsystems and the constraint equations (1) and (2) are obtained in the form

$$
M_{r}=\left[\begin{array}{llllllllllllll}
3 & 0 & 0 & 0 & 0 & 0 & 0 & 0 & 0 & 0 & 0 & 0 & 0 & 0 \\
0 & 1 & 0 & 0 & 0 & 0 & 0 & 0 & 0 & 0 & 0 & 0 & 0 & 0 \\
0 & 0 & 3 & 0 & 0 & 0 & 0 & 0 & 0 & 0 & 0 & 0 & 0 & 0 \\
0 & 0 & 0 & 2 & 0 & 0 & 0 & 0 & 0 & 0 & 0 & 0 & 0 & 0 \\
0 & 0 & 0 & 0 & 4 & 0 & 0 & 0 & 0 & 0 & 0 & 0 & 0 & 0 \\
0 & 0 & 0 & 0 & 0 & 1 & 0 & 0 & 0 & 0 & 0 & 0 & 0 & 0 \\
0 & 0 & 0 & 0 & 0 & 0 & 2 & 0 & 0 & 0 & 0 & 0 & 0 & 0 \\
0 & 0 & 0 & 0 & 0 & 0 & 0 & 2 & 0 & 0 & 0 & 0 & 0 & 0 \\
0 & 0 & 0 & 0 & 0 & 0 & 0 & 0 & 3 & 0 & 0 & 0 & 0 & 0 \\
0 & 0 & 0 & 0 & 0 & 0 & 0 & 0 & 0 & 0 & 0 & 0 & 0 & 0 \\
0 & 0 & 0 & 0 & 0 & 0 & 0 & 0 & 0 & 0 & 0 & 0 & 0 & 0 \\
0 & 0 & 0 & 0 & 0 & 0 & 0 & 0 & 0 & 0 & 0 & 0 & 0 & 0 \\
0 & 0 & 0 & 0 & 0 & 0 & 0 & 0 & 0 & 0 & 0 & 0 & 0 & 0 \\
0 & 0 & 0 & 0 & 0 & 0 & 0 & 0 & 0 & 0 & 0 & 0 & 0 & 0
\end{array}\right] ;
$$

$$
G_{r}=\left[\begin{array}{cccccccccccccc}
1 & -1 & 0 & 0 & 0 & 0 & 0 & 0 & 0 & 1 & 0 & 0 & 0 & 0 \\
-1 & 2 & -1 & 0 & 0 & 0 & 0 & 0 & 0 & 0 & 0 & 1 & 1 & 0 \\
0 & -1 & 1 & 0 & 0 & 0 & 0 & 0 & 0 & 0 & 1 & 0 & 0 & 0 \\
0 & 0 & 0 & 2 & -2 & 0 & 0 & 0 & 0 & 0 & 0 & 0 & 0 & 0 \\
0 & 0 & 0 & -2 & 4 & -2 & 0 & 0 & 0 & 0 & 0 & -1 & 0 & 1 \\
0 & 0 & 0 & 0 & -2 & 2 & 0 & 0 & 0 & 0 & -1 & 0 & 0 & 0 \\
0 & 0 & 0 & 0 & 0 & 0 & 3 & -3 & 0 & -1 & 0 & 0 & 0 & 0 \\
0 & 0 & 0 & 0 & 0 & 0 & -3 & 6 & -3 & 0 & 0 & 0 & -1 & -1 \\
0 & 0 & 0 & 0 & 0 & 0 & 0 & -3 & 3 & 0 & 0 & 0 & 0 & 0 \\
1 & 0 & 0 & 0 & 0 & 0 & -1 & 0 & 0 & -1 & 0 & 0 & 0 & 0 \\
0 & 0 & 1 & 0 & 0 & -1 & 0 & 0 & 0 & 0 & -2 & 0 & 0 & 0 \\
0 & 1 & 0 & 0 & -1 & 0 & 0 & 0 & 0 & 0 & 0 & 0 & 0 & 0 \\
0 & 1 & 0 & 0 & 0 & 0 & 0 & -1 & 0 & 0 & 0 & 0 & 0 & 0 \\
0 & 0 & 0 & 0 & 1 & 0 & 0 & -1 & 0 & 0 & 0 & 0 & 0 & 0
\end{array}\right] .
$$

We normalize the matrix $M_{r}$ of the model (4) by transforming the coordinates in the form

$$
X_{r}=P_{m} X, P_{m}=M_{0}^{-0,5} \oplus E_{4} .
$$

In the coordinate space $X$ model (4) will have the form

$$
M_{m} \ddot{X}+G_{m} X=0,
$$

where $M_{m}=E_{9} \oplus 0_{4}$

$$
\begin{gathered}
G_{m}=\left[\begin{array}{ccc}
G_{0 m} & D_{1 m} & D_{2 m} \\
D_{1 m}^{*} & \Phi & 0_{2} \\
D_{2 m}^{*} & 0 & 0_{2}
\end{array}\right] ; \\
G_{0 m}=M_{0}^{-0,5} G_{0} M_{0}^{-0,5} ; \\
D_{1 m}=M_{0}^{-0,5} D_{1} ; \\
D_{2 m}=M_{0}^{-0,5} D_{2} .
\end{gathered}
$$

Finding the solution of the differential equation (35) in the form $X=\mathrm{H} e^{\lambda t}$ leads to the necessity of solving the generalized eigenvalue problem for the matrix beam $\left(G_{m}, M_{m}\right)$

$$
G_{m} H=\lambda M_{m} H,
$$

where $(\lambda, H)$ - is the proper pair of the beam.

The matrix beam $\left(G_{m}, M_{m}\right)$ has the structure of a singular matrix beam (2) and is characterized by the following numerical content of its components

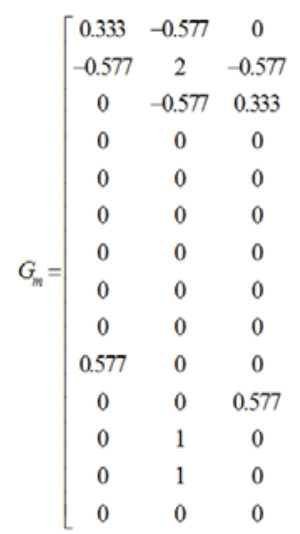




$$
M_{m}=\left[\begin{array}{llllllllllllll}
1 & 0 & 0 & 0 & 0 & 0 & 0 & 0 & 0 & 0 & 0 & 0 & 0 & 0 \\
0 & 1 & 0 & 0 & 0 & 0 & 0 & 0 & 0 & 0 & 0 & 0 & 0 & 0 \\
0 & 0 & 1 & 0 & 0 & 0 & 0 & 0 & 0 & 0 & 0 & 0 & 0 & 0 \\
0 & 0 & 0 & 1 & 0 & 0 & 0 & 0 & 0 & 0 & 0 & 0 & 0 & 0 \\
0 & 0 & 0 & 0 & 1 & 0 & 0 & 0 & 0 & 0 & 0 & 0 & 0 & 0 \\
0 & 0 & 0 & 0 & 0 & 1 & 0 & 0 & 0 & 0 & 0 & 0 & 0 & 0 \\
0 & 0 & 0 & 0 & 0 & 0 & 1 & 0 & 0 & 0 & 0 & 0 & 0 & 0 \\
0 & 0 & 0 & 0 & 0 & 0 & 0 & 1 & 0 & 0 & 0 & 0 & 0 & 0 \\
0 & 0 & 0 & 0 & 0 & 0 & 0 & 0 & 1 & 0 & 0 & 0 & 0 & 0 \\
0 & 0 & 0 & 0 & 0 & 0 & 0 & 0 & 0 & 0 & 0 & 0 & 0 & 0 \\
0 & 0 & 0 & 0 & 0 & 0 & 0 & 0 & 0 & 0 & 0 & 0 & 0 & 0 \\
0 & 0 & 0 & 0 & 0 & 0 & 0 & 0 & 0 & 0 & 0 & 0 & 0 & 0 \\
0 & 0 & 0 & 0 & 0 & 0 & 0 & 0 & 0 & 0 & 0 & 0 & 0 & 0 \\
0 & 0 & 0 & 0 & 0 & 0 & 0 & 0 & 0 & 0 & 0 & 0 & 0 & 0
\end{array}\right]
$$

Using the standard program svb of the MATLAB system, we find the matrix components of the singular decomposition of the submatrix $D_{2 m}=U S V^{\prime \prime}$

where $U, V$ - the left and right singular matrices of the submatrix $D_{2 m} ; S-$ syngular core of the submatrix $D_{2 m}$ :

$$
\begin{aligned}
& U=\left[\begin{array}{ccccccccc}
0 & 0 & -0.792 & 0 & 0 & 0 & 0 & -0.61 & 0 \\
-0.898 & -0.225 & -0.231 & 0 & 0 & 0 & 0 & 0.3 & 0 \\
0 & 0 & 0 & 0 & -1 & 0 & 0 & 0 & 0 \\
0 & 0 & 0 & 1 & 0 & 0 & 0 & 0 & 0 \\
0.159 & 0.635 & 0.461 & 0 & 0 & 0 & 0 & 0.599 & 0 \\
0 & 0 & 0 & 0 & 0 & 1 & 0 & 0 & 0 \\
0 & 0 & 0 & 0 & 0 & 0 & 1 & 0 & 0 \\
0.41 & -0.739 & -0.326 & 0 & 0 & 0 & 0 & 0.424 & 0 \\
0 & 0 & 0 & 0 & 0 & 0 & 0 & 0 & 1
\end{array}\right] ; \\
& S^{*}=\left[\begin{array}{cccccccccc}
1.553 & 0 & 0 & 0 & 0 & 0 & 0 & 0 & 0 \\
0 & 1.043 & 0 & 0 & 0 & 0 & 0 & 0 & 0 \\
0 & 0 & 0 & 0 & 0 & 0 & 0 & 0 & 0
\end{array}\right] ;
\end{aligned}
$$

$$
V=\left[\begin{array}{ccc}
-0.7 & -0.52 & -0.577 \\
-0.765 & 0.285 & 0.577 \\
-0.136 & 0.805 & -0.577
\end{array}\right]
$$

We define the content leading submatrix $\sigma$ of the matrix $S$ in the form:

$$
\sigma=\left[\begin{array}{cc}
1.553 & 0 \\
0 & 1.043
\end{array}\right] .
$$

Following the dependences (4) and (5), we perform the transformation of the coordinates of the model (35) by the formula:

$$
X=P_{s} Y \text { : }
$$

and we represent this model in the form:

$$
M \ddot{Y}+G Y=0 \text {, }
$$

where $P_{s}=U \oplus E_{2} \oplus V$;

$$
\begin{aligned}
& G=\left[\begin{array}{c:c:c}
U^{*} G_{0 m} U & U^{*} D_{1 m} & U^{*} D_{2 m} V \\
\hdashline D_{1 m}^{*} U & \Phi & 0_{2} \\
\hdashline V^{*} D_{2 m}^{*} U & 0_{2} & 0_{2}
\end{array}\right] ; \\
& M=\left[\begin{array}{c:c:c}
E_{9} & 0 & 0 \\
\hdashline 0 & 0 & 0 \\
\hdashline 0 & 0 & 0 \\
0 & & 3
\end{array}\right] .
\end{aligned}
$$

The numerical value of the matrix $G$ is obtained in the form

$$
G=\left[\begin{array}{cc}
2.143 & -0.404 \\
-0.404 & 2.143 \\
-0.471 & 0.431 \\
-0.113 & -0.449 \\
-0.519 & -0.13 \\
-0.159 & -0.635 \\
-0.615 & 1.108 \\
-0.238 & -0.773 \\
-0.502 & 0.905 \\
0 & 0 \\
0 & 0 \\
1.553 & 0 \\
0 & 1.043 \\
0 & 0
\end{array}\right.
$$

$$
\left.\begin{array}{ccc}
1.553 & 0 & 0 \\
0 & 1.043 & 0 \\
0 & 0 & 0 \\
0 & 0 & 0 \\
0 & 0 & 0 \\
0 & 0 & 0 \\
0 & 0 & 0 \\
0 & 0 & 0 \\
0 & 0 & 0 \\
0 & 0 & 0 \\
0 & 0 & 0 \\
0 & 0 & 0 \\
0 & 0 & 0 \\
0 & 0 & 0
\end{array}\right] .
$$

The submatrix $U^{*} D_{2 m} V=S$ of the model under consideration has an incomplete rank, which generates a common zero-space for the components of the calculated matrix beam $(G, M)$, which should be excluded. This exception occurs as a result of deleting the last row and the last column of the matrices $G$ and $M$. The corresponding transformation of the model (38) is determined by the dependencies:

$$
\begin{aligned}
& Y=P_{\Delta} Q ; \\
& \bar{M} \ddot{Q}+\bar{G} Q=0 ; \\
& \bar{M}=P_{\Delta}^{*} M P_{\Delta} ; \\
& \bar{G}=P_{\Delta}^{*} G P_{\Delta} ; \\
& P_{\Delta}=\left[\begin{array}{c}
E_{13} \\
0_{1,13}
\end{array}\right] .
\end{aligned}
$$


The singular spectral analogue of the conservative model (39) will have the form:

$$
\bar{G} Z=\lambda \bar{M} Z,
$$

where $(\lambda, Z)$ - the intrinsic pair of the matrix beam $(\bar{G}, \bar{M})$

The numerical fields of the obtained components of the matrix beam $(\bar{G}, \bar{M})$ are segmented, following the scheme (12)
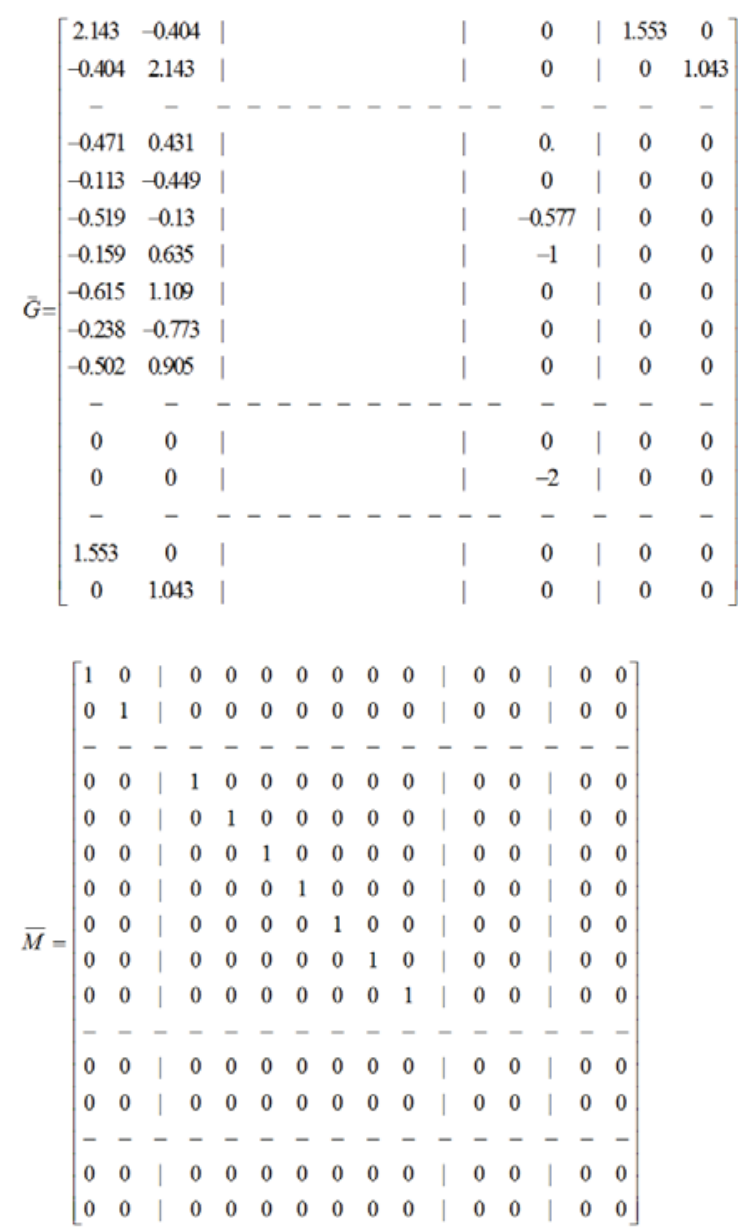

In accordance with the segmentation of the matrix beam $(\bar{G}, \bar{M})$, the symbolic representation of the spectral equation (40) is taken in the form:

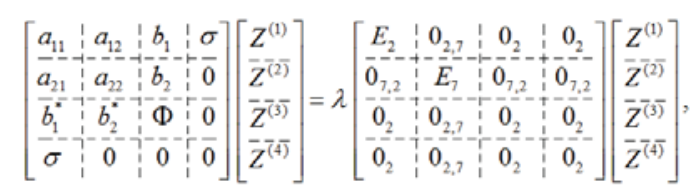

where $Z^{(i)},(i=1,2,3,4)$ - the tuples of the eigenvector $Z$ that are structurally consistent with the matrix beam $(G$, M) segmentation scheme.

Solving the matrix equations corresponding to the four horizontal segments of the system (40), from the bottom to the top, we obtain

$$
\left.\begin{array}{c}
\sigma Z^{(1)}=0 \Rightarrow Z^{(1)}=0 ; \\
b_{2}^{*} Z^{(2)}+\Phi Z^{(3)}=0 \Rightarrow Z^{(3)}=-\Phi^{-1} b_{2}^{*} Z^{(2)} ; \\
\left(a_{22}-b_{2} \Phi^{-1} b_{2}^{*}\right) Z^{(2)}=\lambda Z^{(2)} \Rightarrow\left(a_{22}-b_{2} \Phi^{-1} b_{2}^{*}-\lambda E_{7}\right) Z^{(2)}=0 ; \\
a_{12} Z^{(2)}+b_{1} Z^{(3)}+\sigma Z^{(4)}=0 \Rightarrow Z^{(4)}=-\sigma^{-1}\left(a_{12} Z^{(2)}+b_{1} Z^{(3)}\right) .
\end{array}\right\}
$$

It follows from the expression in the third line of system (42) that the 7 final eigenvalues of a symmetric calculated beam $(G, \Theta)$ can be found as a result of solving the standard eigenvalue problem for a symmetric matrix $A=a_{22}-b_{2} \Phi^{-1} b_{2}^{\prime \prime}$. In accordance with the segmentation of the matrix $G$ performed, the numerical value of the matrix $A$ is defined as

$$
A=\left[\begin{array}{ccccccc}
0.636 & 0.326 & -0.133 & 0.461 & 0.489 & -0.612 & 0.399 \\
0.326 & 1 & 0 & 0 & 0 & -0.424 & 0 \\
-0.133 & 0 & 0.333 & 0 & 0 & 0.173 & 0 \\
0.461 & 0 & 0 & 2 & 0 & -0.599 & 0 \\
0.489 & 0 & 0 & 0 & 1.5 & -0.635 & 0 \\
-0.612 & -0.424 & 0.173 & -0.599 & -0.635 & 1.411 & -0.519 \\
0.399 & 0 & 0 & 0 & 0 & -0.519 & 1
\end{array}\right] .
$$

Solving the standard problem of eigenvalues using the standard program MATLAB, we find the matrix of eigenvalues and the modal matrix $\mu_{A}$ of the matrix $A$ :

$$
\begin{aligned}
& S P=\operatorname{diag}\left(\begin{array}{lllllll}
0 & 0.488 & 0.683 & 1 & 1.606 & 2.33 & 3.274
\end{array}\right) \\
& \mu_{A}=\left[\begin{array}{ccccccc}
0.652 & 0.427 & -0.43 & 0 & 0 & 0.232 & 0.393 \\
-0.309 & -0.079 & -0.367 & 0.775 & 0.368 & 0.0819 & 0.148 \\
0.378 & -0.843 & -0.321 & 0 & -0.161 & -0.132 & 0.0151 \\
-0.218 & 0.0925 & -0.0394 & 0 & -0.301 & -0.682 & 0.622 \\
-0.309 & -0.17 & 0.0878 & 0 & -0.517 & 0.664 & 0.4 \\
-0.227 & 0.233 & -0.606 & 0 & -0.527 & -0.0784 & -0.494 \\
-0.378 & -0.968 & -0.449 & -0.633 & 0.451 & 0.1 & 0.182
\end{array}\right]
\end{aligned}
$$

The eigenvalues of the matrix $A$ are the eigenvalues of the model (38) and the original singular dynamic model (33).

The singular modal matrix $\mu_{z}$ of the matrix beam $(\vec{G}, \bar{M})$ of the model (40) can be defined as the vertical concatenation of four matrices $\mu_{z 1}, \mu_{z 2}, \mu_{z 3}, \mu_{z 4}$ related to each other by the same relations as the tuples $Z^{(1)}, Z^{(2)}, Z^{(3)}, Z^{(4)}$ of the eigenvector $Z$ according to the relations (42):

$$
\mu_{z}=\left(\mu_{z 1}, \mu_{z 2}, \mu_{z 3}, \mu_{z 4}\right)^{*},
$$

$$
\begin{aligned}
& \mu_{z 1}=0_{2,7} ; \\
& \text { where } \mu_{z 2}=\mu_{A} \text {; } \\
& \mu_{z 3}=-\Phi^{-1} b_{2}^{*} \mu_{z 2} \text {; } \\
& \mu_{z 4}=-\sigma^{-1}\left(a_{12} \mu_{z 2}+b_{1} \mu_{z 3}\right) \text {; }
\end{aligned}
$$




$$
\mu_{z}=\left[\begin{array}{ccccccc}
0 & 0 & 0 & 0 & 0 & 0 & 0 \\
0 & 0 & 0 & 0 & 0 & 0 & 0 \\
0.652 & 0.427 & -0.43 & 0 & 0 & 0.232 & 0.393 \\
-0.309 & -0.079 & -0.367 & 0.775 & 0.368 & 0.0819 & 0.148 \\
0.378 & -0.843 & -0.321 & 0 & -0.161 & -0.132 & 0.0151 \\
-0.218 & 0.0925 & -0.0394 & 0 & -0.301 & -0.682 & 0.622 \\
-0.309 & -0.17 & 0.0878 & 0 & -0.517 & 0.664 & 0.4 \\
-0.227 & 0.233 & -0.606 & 0 & -0.527 & -0.0784 & -0.494 \\
-0.378 & -0.0968 & -0.449 & -0.633 & 0.451 & 0.1 & 0.182 \\
0 & -0.157 & 0.348 & 0 & 0.551 & -0.548 & -0.289 \\
0 & 0.197 & 0.113 & 0 & 0.197 & 0.379 & -0.315 \\
0 & -0.211 & -0.472 & -0.148 & -0.198 & 0.246 & 0.34 \\
0 & 0.178 & -0.196 & 0.882 & -0.277 & -1.343 & -0.666
\end{array}\right]
$$

The modal matrix $\mu$ of the singular matrix beam $\left(G_{r}, M_{r}\right)$ of the calculating model (33), taking into account the transformations of the coordinates of the model (33), performed in accordance with expressions (34), (38), and (39):

$$
\begin{aligned}
& \mu=P_{m} P_{s} P_{\Delta} \mu_{z}=\left(M_{0}^{-0,5} \oplus E_{4}\right) . \\
& \left(U \oplus E_{2} \oplus V\right)\left[\begin{array}{c}
E_{13} \\
0_{1,13}
\end{array}\right] \mu_{z}= \\
& {\left[\begin{array}{ccccccc}
-0.218 & -0.277 & 0.41 & 0 & 0.186 & -0.0787 & -0.0057 \\
-0.218 & -0.0286 & -0.0822 & 0 & -0.158 & -0.077 & -0.238 \\
-0.218 & 0.487 & 0.186 & 0 & 0.0929 & 0.0761 & -0.0087 \\
-0.218 & -0.0559 & -0.259 & 0.548 & 0.26 & 0.0579 & 0.105 \\
-0.218 & -0.0286 & -0.0822 & 0 & -0.158 & -0.077 & -0.238 \\
-0.218 & 0.0925 & -0.0394 & 0 & -0.301 & -0.682 & 0.622 \\
-0.218 & -0.12 & 0.0621 & 0 & -0.366 & 0.47 & 0.283 \\
-0.218 & -0.0286 & -0.0822 & 0 & -0.158 & -0.077 & -0.238 \\
-0.218 & -0.0559 & -0.259 & -0.365 & 0.26 & 0.0579 & 0.105 \\
0 & -0.157 & 0.348 & 0 & 0.551 & -0.548 & -0.289 \\
0 & 0.197 & 0.113 & 0 & 0.197 & 0.379 & -0.315 \\
0 & 0.0402 & 0.399 & -0.365 & 0.268 & 0.543 & 0.132 \\
0 & 0.212 & 0.305 & 0.365 & 0.0722 & -0.571 & -0.45 \\
0 & 0.172 & -0.0941 & 0.73 & -0.196 & -1.114 & -0583
\end{array}\right]}
\end{aligned}
$$

The modal transformation of the original computational model, accompanied by the transformation of its singular matrix beam $\left(G_{r}, M_{r}\right)$ in the form

$$
G_{E}=\mu^{*} G_{r} \mu ; M_{E}=\mu^{*} M_{r} \mu,
$$

leads the design model to the normal form with a diagonal matrix beam:

$$
\begin{aligned}
& \ddot{R}+G_{R} R=0, \\
& \text { where } R=\mu X_{r} ;
\end{aligned}
$$

\section{EXPERIMENT}

As an experiment or a control calculation, we construct a solution of the generalized eigenvalue problem for a symmetric matrix beam $\left(G_{c}, M_{c}\right)$ corresponding to the differential equation of motion of the dynamical system in question when it is schematized as a free system. The numerical values of the beam components $\left(G_{c}, M_{c}\right)$ in accordance with the equations of constraints (30) and (31) can be represented in the form

$$
\begin{gathered}
G_{c}=\left[\begin{array}{ccccccc}
2.0000 & -1.0000 & 0 & 0 & 0 & -1.0000 & 0 \\
-1.0000 & 12.0000 & -1.0000 & -2.0000 & -2.0000 & -3.0000 & -3.0000 \\
0 & -1.0000 & 1.5000 & 0 & -0.5000 & 0 & 0 \\
0 & -2.0000 & 0 & 2.0000 & 0 & 0 & 0 \\
0 & -2.0000 & -0.5000 & 0 & 2.5000 & 0 & 0 \\
-1.0000 & -3.0000 & 0 & 0 & 0 & 4.0000 & 0 \\
0 & -3.0000 & 0 & 0 & 0 & 0 & 3.0000
\end{array}\right] ; \\
M_{c}=\operatorname{diag}\left(\begin{array}{llllccc}
3 & 7 & 3 & 2 & 1 & 2 & 3
\end{array}\right) .
\end{gathered}
$$

The matrix of eiaenvalues $S \bar{P}$ and the modal matrix $\bar{\mu}$ of the beam $\left(G_{c}, M_{c}\right)$ are calculated by the standard program of the system MATLAB $[\bar{\mu}, S \bar{P}]=\operatorname{eig}\left(G_{c}, M_{c}\right)$ :

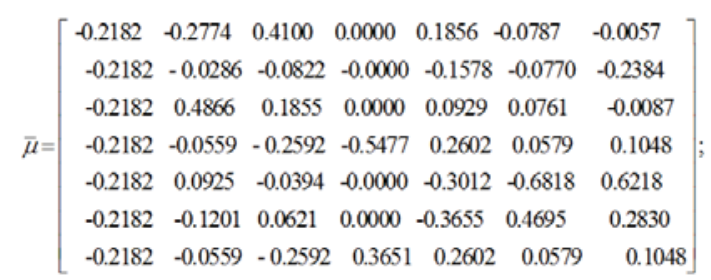

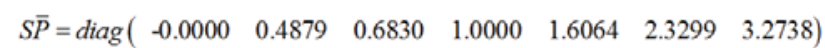

Comparison of the values obtained $\bar{\mu}, S \bar{P}$ according to the expressions (45), (46) and the values $\mu, S P$ according to expressions (44), (43), taking into account the equations of constraints (30) and (31), indicates a correct coincidence of these values.

\section{CONCLUSIONS}

The solution of the generalized problem of eigenvalues and vectors for singular matrix beams opens new horizons for the application of modern mathematical-analytical methods in the field of multicomponent systems with a complex dynamic structure. It is obvious that the promising areas of development presented in this article are methods for introducing soft calculations, fractals, methods of modern mathematical topology, mathematical probability theory, theory of possibilities, etc. into the appropriate mathematical and software applications. The introduction of these applications into a mathematical apparatus solutions of research problems similar to those described in this article is in demand and has been considered in a number of works, examples of which, when edeny in [04-19].

The development and justification of the concretized interpretations of these methods for solving the generalized problem of eigenvalues and vectors for singular matrix beams for new subject domains of investigation constitutes the essence of further work on the devel- 
opment of the reduced mathematical apparatus. At the same time, today we can state a broad perspective and applied applicability of this approach to the development of scientific and methodological tools for analyzing complex multi-component objects.

\section{REFERENCES}

1. Kochura, A., Podkolzina, L., Ivakin, Ya., Nidziev, I. (2013). Singular matrix beams in the generalized symmetric eigenvalue problem. SPIIRAS Proceedings, vol. 26, no. 3, 253-276, DOI: http://dx.doi. org/10.15622/sp.26.18, from http://proceedings. spiiras.nw.ru/ojs/index.php/sp/article/view/1705, accessed on 2017-09-21.

2. Parlett, B. (1998). Symmetric eigenvalue problem. Society for Industrial and Applied Mathematics, Philadelphia, DOI: 10.1137/1.9781611971163

3. Yousef, S. (2003). Iterative methods for sparse linear systems. Society for Industrial and Applied Mathematic, Philadelphia, DOI: 10.1137/1.9780898718003. ch4, from http://inis.jinr.ru/sl/M_Mathematics/MN_ Numerical\%20methods/MNI_Numerical\%20linear\%20algebra/Saad\%20lterative.pdf, accessed on 2017-09-20.

4. Kochura, A., Podkolzina, L., Ivakin, Ya., Nidziev, I. (2014). Development of algorithm of the decision of systems linear equations with the varied parameters, using the matrix sparseness. SPIIRAS Proceedings, no. 2(33), 79-98, DOI: 10.15622/ sp.33.5, from https://readera.ru/razrabotka-algoritma-reshenija-sistem-linejnyh-uravnenij-s-variruemymi-142176937-en, accessed on 2017-08-09.

5. Alaghband, G. (1999). Parallel sparse matrix solution and performance. Parallel Computing, vol. 21, no. 9, 1407-1430, DOI: 10.1016/0167-8191(95)00029-N

6. Blatov, I.A., Kitaeva, Ye.V. (2010). Numerical methods for sparse matrices. Samara State University Publ., Samara.

7. Borutzky, W. (2009). Bond graph methodology: Development and analysis of multidisciplinary dynamic system models. Springer, DOI: 10.1007/978-184882-882-7, from https://link.springer.com/content/ pdf/bfm\%3A978-1-84882-882-7\%2F1.pdf, accessed on 2017-08-22.

8. Dehnavi, M.M., Fernandez, D.M., Giannacopoulos, D. (2010). Finite-element sparse matrix vector multiplication on graphic processing units. IEEE Transactions on Magnetics, vol. 46, no. 8, 2982-2985, DOI: 10.1109/TMAG.2010.2043511

9. Kron, G. (1972). The study of complex systems in parts - diakoptics. Nauka, Moscow.

10. Pissanetski, S. (1988). Sparse matrix technology. Mir, Moscow, from http://bookre.org/reader?file $=1035085$, accessed on 2017-07-05.

11. Sasaoka, T., Kawabata, H., Kitamura, T. (2007). A
MATLAB-based code generator for parallel sparse matrix computations utilizing PSBLAS. IEICE Transactions on Information and Systems, vol. 90-D, no. 1, 2-12, DOI: 10.1093/ietisy/e90-1.1.2

12. Davis, T.A. (2006). Direct methods for sparse linear systems. Society for Industrial and Applied Mathematic, New York, DOI: 10.1111/j.17515823.2007.00015_14.X

13. Davis, T.A., Hu, Y. (2011). The university of Florida sparse matrix collection. ACM Transactions on Mathematical Software, vol. 38, no. 1, 1-28, DOI: 10.1145/2049662.2049663, from http://yifanhu.net/ PUB/matrices.pdf, accessed on 2017-08-25.

14. Tran, T.M., Gruber, R., Appert, K., Wuthrich, S. (1996). A direct parallel sparse matrix solver. Computer Physics Communications, vol. 96, no. 2-3, 118-128, DOI: 10.1016/0010-4655(96)00007-0

15. Manakova, N.A., Vasiuchkova, K.V. (2017). Numerical investigation for the start control and final observation problem in model of an i-beam deformation. Journal of Computational and Engineering Mathematics. vol. 4, no. 2. 26-40, DOI: 10.14529/ jcem170203. MSC 35Q99

16. Al-Isawi, J.K.T. (2015). On some properties of solutions to dzektser mathematical model in quasi-sobolev spaces. Journal of Computational and Engineering Mathematics. vol. 2, no. 4. 27-36. DOI: 10.14529/jcem150403. MSC 46A16, 47D03, 34D09

17. Ivakin, Ya., Potapychev, S.N., Ivakin, V. (2018). Verification of the hypotheses of a research on the basis of geochronological tracking. Historical Informatics. vol.1. 86-93 DOI: 10.7256/2585-7797.2018.1.25344. URL: http://e-notabene.ru/istinf/article_25344.html

18. Uteshev, A.U., Borovoj I.I. (2016) The solution of a problem of rational interpolation with use of gankelevy polynoms. Vestnik of Saint Petersburg University. Ser. 10, Applied Mathematics. Computer Science. Control Processes. vol. 4, 31-43 DOI: 10.21638/11701/ spbu10.2016.403

19. Aliseyko, A.N. (2017). Lyapunov's matrixes for a class of systems with an exponential kernel. Vestnik of Saint Petersburg University. Ser. 13, Applied Mathematics. Computer Science. Control Processes. vol. 3, 228-240 DOI: 10.21638/11701/spbu10.2017.301

Paper submitted: 06.03.2018.

Paper accepted: 22.05.2018.

This is an open access article distributed under the CC BY-NC-ND 4.0 terms and conditions. 